\title{
Über die Einwirkung von Natriumalkoholat auf Acetessigester
}

\author{
von \\ Dr. Telemachos Komnenos, \\ Privatdozent der Chemie.
}

Aus dem Dambergi'schen pharm.-chemischen Laboratorium der Universität zu Athen.

(Vorgelegt in der Sitzung am 21. April 1910.)

In einer früheren Abhandlung ${ }^{1}$ bewies ich, daß bei der Einwirkung von in Methylalkohol gelöstem Natriummethylat auf Äthylmalonat die Äthylgruppe des Esters durch die Methylgruppe ersetzt wird und behielt mir vor, diese Methode der Alkylvertretbarkeit auch bei anderen Estern anzuwenden. Nun fing ich zunächst mit dem Acetylessigsäureester an.

\section{Versuch mit Acetylessigsäureäthylester und Natrium- methylat.}

$23 \mathrm{~g}$ Natrium wurden in zirka $300 \mathrm{~cm}^{3}$ Methylalkohol gelöst und die noch warme Flüssigkeit mit $130 \mathrm{~g}$ Acetylessigsäureäthylester gemischt, wobei sich kein Niederschlag bildete. Nach 24 Stunden wurde die Mischung mit viel Wasser verdünnt, dann mit Salzsäure eingesäuert und zweimal mit je $200 \mathrm{~cm}^{3}$ Äther geschüttelt. Die Ätherschichten wurden vereinigt und nach Destillieren des Äthers der Rückstand fraktioniert. Es wurden so folgende Fraktionen erhalten:

bis $120^{\circ} \ldots \ldots 12 \mathrm{~cm}^{3}$,

120 bis $155^{\circ} \ldots \ldots 7 \cdot 5 \mathrm{~cm}^{3}$,

155 bis $165^{\circ} \ldots \ldots 36 \mathrm{~cm}^{3}$,

165 bis $172^{\circ} \ldots \ldots 6 \mathrm{~cm}^{3}$.

1 Über die Vertretbarkeit der in den Säureestern befindlichen Alkyle. Monatshefte für Chemie 1910, 111. 
Der Acetylessigsäureäthylester siedet bekanntlich bei $181^{\circ}$, folglich enthält das Reaktionsprodukt keine Spur davon. Die Fraktionen 155 bis $165^{\circ}$ und 165 bis $172^{\circ}$ wurden wieder destilliert und das zwischen 167 bis $169^{\circ}$ Übergehende separat für die Untersuchung gesammelt.

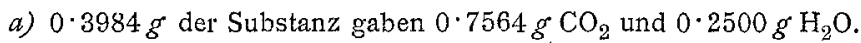

b) $0.2643 \mathrm{~g}$ der Substanz gaben $0.6743 \mathrm{~g} \mathrm{CO}_{2}$ und $0.1660 \mathrm{~g} \mathrm{H}_{2} \mathrm{O}$.

Prozentische Zusammensetzung:

\begin{tabular}{|c|c|c|}
\hline & Iden & Berechnet für \\
\hline$a$ & $b$ & \\
\hline$\ldots 51 \cdot 77$ & $51 \cdot 51 \%$ & $51 \cdot 72 \%$ \\
\hline $\mathrm{H} \ldots \ldots \ldots 6.98$ & $6 \cdot 97$ & $6 \cdot 89$ \\
\hline $0 \ldots \ldots \ldots 41 \cdot 25$ & $41 \cdot 52$ & $41 \cdot 39$ \\
\hline $100 \cdot 00$ & $100 \cdot 00$ & $100 \cdot 00$ \\
\hline
\end{tabular}

$0.285 \mathrm{~g}$ der Substanz, in $10.630 \mathrm{~g}$ absolutem Alkohol gelöst, erhöhten den Siedepunkt desselben um $0.25^{\circ}$.

Molekulargewicht... $\underbrace{\text { Gefunden }}_{125} \quad \underbrace{\begin{array}{c}\text { Berechnet für } \\ \mathrm{C}_{5} \mathrm{H}_{8} \mathrm{O}_{3}\end{array}}_{116}$

Dann wurde das spezifische Gewicht des Körpers $\mathrm{C}_{5} \mathrm{H}_{8} \mathrm{O}_{3}$ bestimmt und bei $10^{\circ} \mathrm{C} .1 .0368$ gefunden. Mit Eisenchlorid erzeugt der Ester eine dunkle, kirschrote Färbung; während der Äthylester eine violette bildet.

Aus allen diesen Ergebnissen geht hervor, daß der Körper $\mathrm{C}_{5} \mathrm{H}_{8} \mathrm{O}_{2}$ der Acetylessigsäuremethylester ist und daß die Umwandlung des Äthylesters in den Methylester eine fast quantitative ist, da bei $172^{\circ}$ der Destillationskolben nichts mehr enthielt.

Der Methylacetessigester, dargestellt von Brandes ${ }^{1}$ aus Methylacetat und Natrium, siedet bei $169^{\circ}$ und hat das spezifische Gewicht 1.037 bei $9^{\circ}$.

Es hinterliegt mithin kein Zweitel, daß auch bei diesem Falle die Äthylgruppe des Acetylessigesters durch die Methylgruppe völlig ersetzt wurde nach der Gleichung

$$
\begin{array}{r}
\mathrm{CH}_{3}-\mathrm{CO}-\mathrm{CH}_{2}-\mathrm{COOC}_{2} \mathrm{H}_{5}+\mathrm{NaOCH}_{3}= \\
=\mathrm{CH}_{3}-\mathrm{CO}-\mathrm{CH}_{2}-\mathrm{COOCH}_{3}+\mathrm{NaOC}_{2} \mathrm{H}_{5} .
\end{array}
$$

2 Zeitschr. für Chemie, 1866, 454. 
Sehr bemerkenswert ist, daß bei der Destillation des Methylacetessigesters keine Dehydracetsäurebildung wahrnehmbar ist.

Um festzustellen, ob die Reaktion auch rückwärts gehen kann, unternahm ich folgenden Versuch.

\section{Versuch mit Acetylessigsäuremethylester und Natrium- äthylat.}

$\mathrm{Zu}$ dem wie oben dargestellten Methylacetessigester $(100 \mathrm{~g})$ wurde die entsprechende Menge Natriumäthylat (auf $1 \mathrm{Mol}$ Ester 1 Atom Natrium) zugesetzt und im übrigen dasselbe Verfahren angewendet, wie oben beschrieben. Bei derDestillation wurden folgende Fraktionen erhalten:

$$
\begin{array}{r}
\text { bis } 120^{\circ} \ldots \ldots 13 \mathrm{~cm}^{3} \text {, } \\
120 \text { bis } 155^{\circ} \ldots \ldots 6 \mathrm{~cm}^{3} \text {, } \\
155 \text { bis } 175^{\circ} \ldots \ldots 10 \mathrm{~cm}^{3} \text {, } \\
175 \text { bis } 185^{\circ} \ldots \ldots 40 \mathrm{~cm}^{3} \text {. }
\end{array}
$$

Über $185^{\circ}$ ging eine kleine Menge Dehydracetsäure über, welche teilweise in dem Kühler erstarrte und bei $109^{\circ} \mathrm{schmolz}$.

Die Fraktion 175 bis $185^{\circ}$ wurde noch zweimal destilliert und das bei 180 bis $182^{\circ}$ Übergehende separat gesammelt. Dasselbe zeigt das spezifische Gewicht 1.025 bei $20^{\circ} \mathrm{C}$. (der Acetessigsäureäthylester hat nach $\mathrm{Br}$ ühl das spezifische Gewicht 1.0256 bei $20 \cdot 4^{\circ}$ ), gibt mit Eisenchlorid eine violette Färbung und besteht jedenfalls aus Acetessigsäureäthylester $\mathrm{CH}_{3}-\mathrm{CO}-$ $\mathrm{CH}_{2}-\mathrm{COOC}_{2} \mathrm{H}_{5}$. Seine weitere Untersuchung zur Feststellung der Identität wurde für überflüssig gehalten.

- Mithin ist bewiesen wie auch bei früheren Fällen, ${ }^{1}$ daß die Reaktion umkehrbar ist, d. h. der Äthylester wird durch Natriummethylat in den Methylester verwandelt und der letztere durch Natriumäthylat bildet wieder den Äthylester nach der Gleichung:

$$
\begin{array}{r}
\mathrm{CH}_{3}-\mathrm{CO}-\mathrm{CH}_{2}-\mathrm{COOC}_{2} \mathrm{H}_{5}+\mathrm{NaOCH}_{3} \rightleftarrows \\
\mathrm{NaOC}_{2} \mathrm{H}_{5}+\mathrm{CH}_{3}-\mathrm{CO}-\mathrm{CH}_{2}-\mathrm{COOCH}_{3} .
\end{array}
$$

1 Monatshefte für Chemie, 1910, 119. Aus Acetylentetracarbonsäuremethylester wurde der Äthylester dargestellt und umgekehrt. 


\section{Versuch mit Acetylessigsäureäthylester und Natrium- amylat.}

$11.5 \mathrm{~g}$ Natrium wurden in kleine Stücke geschnitten und in zirka $150 \mathrm{~cm}^{3}$ Amylalkohol gelöst. ${ }^{1}$ In die noch warme Natriumamylatlösung wurden in kleinen Portionen $65 \mathrm{~g}$ Acetylessigsäureäthylester zugesetzt. Es fand eine heftige Reaktion statt, die Flüssigkeit färbte sich beim ersten Esterzusatz rötlich und fing zu kochen an, so daß beim Zusatz der späteren Portionen die Flasche durch kaltes Wasser gekühlt und mit 'dem Rückflußkühler verbunden wurde. Gleichzeitig machte sich ein eigentümlicher birnenartiger Geruch bemerkbar. Nach zwei Stunden war der Inhalt der Flasche fest. Am folgenden Tage wurde die Masse in $200 \mathrm{~cm}^{3}$ Wasser gelöst und mit Salzsäure angesäuert, wobei sich eine reichliche Ölschicht schied, welche aber nicht ganz durchsichtig war. Es wurde deshalb die ganze Flüssigkeit mit zirka $150 . \mathrm{cm}^{3}$ Äther geschüttelt, die Ätherschicht über Chlorcalcium getrocknet und am folgenden Tage einer fraktionierten Destillation unterworfen. Nach dem Überdesti1lieren des Äthers wurden folgende Fraktionen erhalten:

120 bis $140^{\circ} \ldots \ldots 66 \mathrm{~cm}^{3}$, Geruch nach Amylalkohol, 140 bis $160^{\circ} \ldots \ldots 20 \mathrm{~cm}^{3}$,,$\quad$, etwas esterartig,

160 bis $210^{\circ} \ldots \ldots 10 \mathrm{~cm}^{3}$, Geruch esterartig, 210 bis $230^{\circ} \ldots \ldots .60 \mathrm{~cm}^{3}$, 》 230 bis $240^{\circ} \ldots \ldots 15 \mathrm{~cm}^{3}$, " empyreumatisch.

Am nächsten Tage war die Fraktion 230 bis $240^{\circ}$ halbfest. Es schieden sich reichlich weiße Kryställchen, welche bei $109^{\circ}$ schmolzen. Diese wurden filtriert und zur weiteren Untersuchung beiseite gestellt. Bei der zweiten Destillation wurden folgende Fraktionen erhalten:

1 Die Reaktion war am Anfange sehr schwach. Die Flüssigkeit wurde gelb, dann erwärmte sie sich von selbst, die gelbe Farbe verschwand fast ganz und das Natrium bildete eine grofe geschmolzene Kugel, welche heftig Wasserstoff entwickelte. Dann ließ die Reaktion nach und ein neuer Amylalkoholzusatz sowie Erwärmung auf dem Wasserbade half wenig. Nun wurde die Flasche vorsichtig auf dem Drahtnetze eine Stunde lang bis zum Siedepunkt des Amylalkohols erwärmt und war so alles Natrium aufgelöst. 
120 bis $140^{\circ} \ldots \ldots .80 \mathrm{~cm}^{3}$,
140 bis $160^{\circ} \ldots \ldots 5 \mathrm{~cm}^{3}$,
160 bis $210^{\circ} \ldots \ldots 10 \mathrm{~cm}^{3}$,
210 bis $220^{\circ} \ldots \ldots 20 \mathrm{~cm}^{3}$,
220 bis $230^{\circ} \ldots \ldots 15 \mathrm{~cm}^{3}$,
230 bis $240^{\circ} \ldots \ldots 5 \mathrm{~cm}^{3}$.

Die Fraktion 230 bis $240^{\circ}$ wurde sogleich fest und die ausgeschiedenen Krystalle schmolzen bei $109^{\circ}$. Da ich aus Erfahrung das Aussehen dieser bei der Destillation des Acetylessigsäureäthylesters sich immer bildenden Krystalle von Dehydracetsäure gut kenne, war ich sofort der Meinung, zumal der Schmelzpunkt stimmt, daß es sich um diese Säure handelt, bewahrte jedoch die feste Masse zur weiteren Untersuchung, wie weiter unten angegeben wird.

Untersuchung der bei 220 bis $230^{\circ}$ (konstant bei $225^{\circ}$ ) siedenden Fraktion.

Dieselbe ist eine schwach gelbliche Flüssigkeit, siedet fast konstant bei $225^{\circ}$, hat einen eigentümlich aromatischen, an Amylacetat erinnernden Geruch, bei $10^{\circ} \mathrm{C}$. hat sie das spezifische Gewicht $0 \cdot 954$ und bei jeder Destillation verwandelt sie sich zum großen Teil in eine feste, krystallinische Substanz, welche die Fraktion 230 bis $240^{\circ}$ zum größten Teil bildet.

Um diese Flüssigkeit möglichst rein für die Analyse zu haben, setzte ich die Fraktion 220 bis $230^{\circ}$ in ein kleines Fraktionskölbchen, destillierte es rasch durch direkte Erhitzung mit der Flamme, also ohne Drahtnetz und sammelte separat den bei $225^{\circ}$ konstant siedenden Anteil.

$0.234 \mathrm{~g}$ der Substanz lieferten $0.5367 \mathrm{~g} \mathrm{CO}_{2}$ und $0.200 \mathrm{~g} \mathrm{H}_{2} \mathrm{O}$.

Prozentische Zusammensetzung:

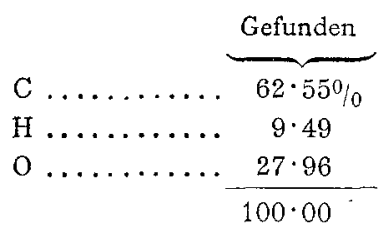

$$
\begin{gathered}
\begin{array}{c}
\text { Berechnet für } \\
\mathrm{C}_{9} \mathrm{H}_{16} \mathrm{O}_{3}
\end{array} \\
\hline 62 \cdot 79 \% \\
9 \cdot 30 \\
\frac{27 \cdot 91}{100 \cdot 00}
\end{gathered}
$$


$0 \cdot 2356 \mathrm{~g}$ der Substanz, in $13 \cdot 250 \mathrm{~g}$ absolutem Alkohol gelöst, erhöhten den

Siedepunkt desselben um $0 \cdot 12^{\circ}$.

Molekulargewicht. . $\underbrace{\text { Gefunden }}_{173 \cdot 3} \quad \underbrace{\mathrm{C}_{9} \mathrm{H}_{16} \mathrm{O}_{3}}_{172}$

Aus diesen Zahlen ergibt sich, daß der bei $225^{\circ}$ siedende Körper der Amylacetessigester $\mathrm{CH}_{3}-\mathrm{CO}-\mathrm{CH}_{2}-\mathrm{COOC}_{5} \mathrm{H}_{11}$ ist, welchen Conrad ${ }^{1}$ aus Isoamylacetat und Natrium dargestellt, und welcher nach seiner Angabe bei $223^{\circ}$ siedet und bei $10^{\circ}$ das spezifische Gewicht 0.954 hat. Folglich ist der Ersatz der Gruppe $\mathrm{C}_{2} \mathrm{H}_{5}$ durch die $\mathrm{C}_{5} \mathrm{H}_{11}$, wie voraussichtlich, glatt vor sich gegangen.

Untersuchung des aus der Fraktion 230 bis $240^{\circ}$ abgeschiedenen festen Körpers.

Derselbe, aus absolutem Alkohol umkrystallisiert, bildet weiße, unter dem Mikroskop nadelförmige Kryställchen, welche bei $109^{\circ}$ schmelzen, und wurde folgenderweise untersucht. $0.304 \mathrm{~g}$ der Substanz lieferten $0.6354 \mathrm{~g} \mathrm{CO} 2$ und $0.1328 \mathrm{~g} \mathrm{H}_{2} \mathrm{O}$.

Prozentische Zusammensetzung:

\begin{tabular}{|c|c|c|}
\hline & Gefunden & $\mathrm{C}_{8} \mathrm{H}_{8} \mathrm{O}_{4}$ \\
\hline $\mathrm{C}$ & $57 \cdot 00$ & $57 \cdot 14 \%$ \\
\hline$H \ldots \ldots \ldots \ldots$ & $4 \cdot 85$ & $4 \cdot 76$ \\
\hline \multirow[t]{2}{*}{$o \ldots \ldots \ldots$} & $38 \cdot 15$ & $38 \cdot 10$ \\
\hline & $100 \cdot 00$ & $100 \cdot 00$ \\
\hline
\end{tabular}

$0.1764 \mathrm{~g}$, in $12.0 \mathrm{~g}$ absolutem Alkohol gelöst, erhöhten den Siedepunkt desselben um $0 \cdot 1^{\circ}$.

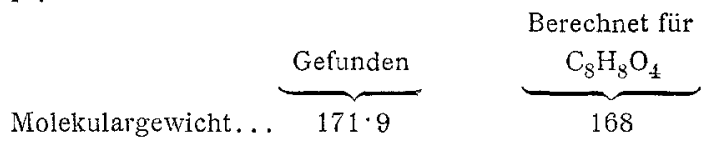

Aus diesen Zahlen ergibt sich, daß die bei $109^{\circ}$ schmelzende Substanz der Fraktion 230 bis $240^{\circ}$, wie vermutet, Dehydracetsäure ist, für welche man jetzt allgemein die Konstitutionsformel<smiles>CC(=O)C1C(=O)OC(C)C=C1C(=O)O</smiles>

1 Annalen der Chemie, 168, 228. 
gibt und welche bekanntlich beim Destillieren des Acetessigesters entsteht, aber nicht in solcher Masse wie hier. Die Konstitutionsformel dieser eigentümlichen Säure, über weiche lange diskutiert wurde, ist nach ihrer Bildung bei der Destillation des Amylacetessigesters nur leicht $z u$ erklären, wenn man annimmt, daß das eine Estermolekül mit seiner Enolform reagiert, und zwar wie folgt:

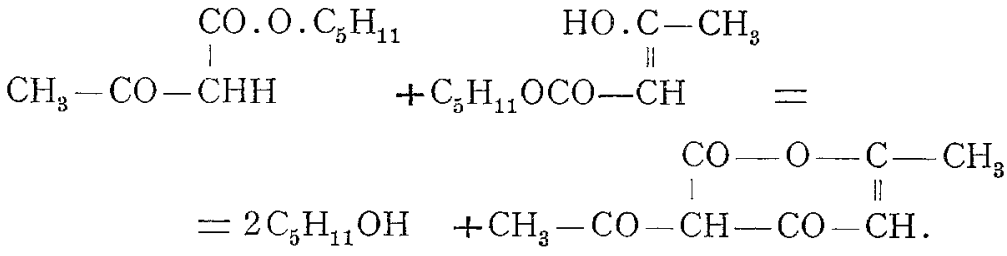

So erklärt sich übrigens auch die bei jeder neuen Destillation bemerkte Vermehrung der Fraktion 120 bis $140^{\circ}$, da sich jedesmal eine neue Quantität Amylalkohol bildete.

Hiermit also wurde bewiesen, daß bei der Einwirkung von Natriumamylat auf $1 \mathrm{Mol}$ Acetessigester sich Amylacetessigester bildet und als Nebenprodukt viel Dehydracetsäure; soviel, daß ich glaube, diese Bildungsweise der so interessanten Säure als die beste Darstellungsmethode empfehlen zu können. 